Revista Brasil. Bot., V.31, n.4, p.597-610, out.-dez. 2008

\title{
Dinâmica da comunidade arbórea de uma mata de galeria do Brasil Central em um período de 19 anos (1985-2004)
}

\author{
ANA PAULA DE OLIVEIRA ${ }^{1}$ e JEANINE MARIA FELFILI ${ }^{2,3}$
}

(recebido: 10 de novembro de 2005; aceito: 29 de agosto de 2008)

\begin{abstract}
Tree community dynamics of a gallery forest in central Brazil over a 19-year period (1985-2004)). Tree community dynamics was analyzed with basis on the data from the continuous inventory in an area of 64 ha of the Gama gallery forest at the Fazenda Água Limpa, DF. The inventory consisted of 151 permanent $(10 \times 20 \mathrm{~m})$ plots located systematically along ten transects placed every $100 \mathrm{~m}$ at right angles to the Gama stream. All individuals with diameter at breast height $(\mathrm{DBH})$ equal or greater than $10 \mathrm{~cm}$ were mapped in the plots, tagged and measured in 1985, 1988, 1991, 1994, 1999 and 2004. The periodic annual increment (PAI) for the community during the 19 years period (1985-2004) was $0.22 \mathrm{~cm} \mathrm{yr}^{-1}$, with coefficients of variation ranging from $84 \%$ to $143 \%$. The trend of increment in diameter was characterized by the increase of the increments with the increasing diameter classes up to $52 \mathrm{~cm}$ of DBH. The mean annual mortality rate was $2.87 \%$ while the recruitment was $2.08 \%$, resulting in a decrease in the density during the 19 years period. The half-life was 24.13 years from 1985 to 2004 . The community was dynamic with $92 \%$ of all species either suffering mortality or gaining recruits or both but, $83 \%$ of total of species found in the forest occurred in all the analyzed periods. Mortality was high but recruitment was also intense leading to a high turnover typical of an environment under strong border effect such as the gallery forests, which are narrow strips of tropical forests surrounded by savanna. These results suggest a very dynamic but stable community.
\end{abstract}

Key words - growth, mortality, recruitment, savanna, tropical forest

RESUMO - (Dinâmica da comunidade arbórea de uma mata de galeria do Brasil Central em um período de 19 anos (19852004)). A dinâmica da comunidade arbórea foi analisada, tendo como base um inventário contínuo em 64 ha da mata de galeria do Gama, na Fazenda Água Limpa, DF. O inventário consistiu de 151 parcelas permanentes $(10 \times 20 \mathrm{~m})$ contíguas locadas em dez transectos eqüidistantes $100 \mathrm{~m}$ uns dos outros. Todos os indivíduos com diâmetro à altura do peito (DAP) maior ou igual a $10 \mathrm{~cm}$ foram mapeados nas parcelas, marcados e medidos em 1985, 1988, 1991, 1994, 1999 e 2004. O incremento periódico anual (IPA) no período de 19 anos foi de $0,22 \mathrm{~cm}^{-1}$ ano ${ }^{-1}$ com coeficientes de variação de $84 \%$ a $143 \%$. $\mathrm{O}$ incremento em diâmetro apresentou tendência crescente com o aumento das classes de diâmetro até $52 \mathrm{~cm}$ de DAP, onde se concentraram aproximadamente $95 \%$ dos indivíduos em cada ocasião. A taxa média anual de mortalidade foi de $2,87 \%$, enquanto o recrutamento foi $2,08 \%$, resultando em um decréscimo na densidade da mata durante os 19 anos de estudo. A meia-vida foi de 24,13 anos de 1985 a 2004. A comunidade foi dinâmica com $92 \%$ de todas as espécies ou sofrendo mortalidade ou ganhando recrutas ou ambos mas, $83 \%$ das espéces ocorreram em todos os períodos analisados. Mortalidade e recrutamento foram elevados, levando a alta rotatividade, típica de ambientes sob intenso efeito de borda como as matas de galeria que são estreitas faixas de floresta tropical, circundadas por savanas. Estes resultados sugerem uma comunidade muito dinâmica mas, estável.

Palavras-chave - crescimento, floresta tropical, mortalidade, recrutamento, savana

\section{Introdução}

As matas de galeria que margeiam os cursos de água no bioma Cerrado (Ribeiro \& Walter 2001) podem ocorrer em terrenos de boa drenagem durante o ano todo assim como em solos estacionalmente inundáveis (Felfili 2001). Um importante aspecto das matas de galeria é a

1. Universidade de Brasília, Departamento de Ecologia, Caixa Postal 4457, 70919-970 Brasília, DF, Brasil.

2. Universidade de Brasília, Laboratório de Manejo Florestal, Departamento de Engenharia Florestal, Caixa Posta 4357, 70919970 Brasília. DF, Brasil.

3._Autor para correspondência: felfili@unb.br sua interface com diversos tipos de vegetação incluindo florestas tropicais úmidas, florestas mesofíticas e cerrado, resultando em uma flora heterogênea e com forte expressão do componente arbóreo (Oliveira-Filho \& Ratter 1995) com espécies endêmicas e algumas em comum com cerrados e florestas (Eiten 1984, OliveiraFilho \& Ratter 1995) distribuindo-se em microsítios determinados por umidade e luz (Felfili 1995 a,b).

Apesar de ocorrerem como pequenas faixas de floresta associadas à umidade em meio a uma matriz de vegetação savânica (Felfili et al. 2001) e serem submetidas a forte efeito de borda, causado por pressões como fortes ventos, fogo, enchentes e queimadas (Felfili 1997a,b, 
Kellman \& Meave 1997), as matas de galeria apresentam elevada riqueza florística (Felfili et al. 2001), e padrões de dinâmica semelhantes àqueles encontrados em florestas tropicais úmidas (Felfili 1995a,b, Felfili 2001).

Na mata do Gama, objeto desse estudo, o incremento médio em diâmetro para um período de seis anos foi de $0,25 \mathrm{~cm} \mathrm{ano}^{-1}$ para a comunidade, sendo mantido um desbalanço de cerca de $1 \%$ a favor da mortalidade (Felfili 1995b). A meia vida prevista para a comunidade da mata do Gama na época foi de 20 anos, sugerindo uma floresta muito dinâmica (Felfili 1995b) quando comparados com outras florestas tropicais. Essas florestas são faixas estreitas rodeadas por ambientes abertos e sujeitas a fortes pressões pelos ventos, enchentes e fogo nas suas bordas (Felfili 1997b) o que justifica a dinâmica intensa verificada para a mata do Gama e do Capetinga no Distrito Federal (Felfili 1995, 1997a,b) e para outras florestas ripárias do Brasil Central (Damasceno-Júnior 2004, Pinto \& Hay 2005, Lopes \& Schiavini 2007). O quanto a heterogeneidade florística e as peculiaridades ambientais que resultam do extenso efeito de borda a que estas florestas estão submetidas (Felfili 1997a,b, Felfili 2001) são expressos nos padrões de dinâmica ainda precisam ser determinado por monitoramentos de longo prazo.

Neste trabalho partiu-se da premissa que a mata de galeria do Gama está em equibilíbrio dinâmico. Espera-se que se confirmem as previsões de Felfili (1995), com base no monitoramento de um período de nove anos (1965-1989), onde espera-se para esta mata, um processo dinâmico intenso mas estável. Conforme a autora, em duas décadas a metade do atual estrato arbóreo seria renovado e o desbalanço encontrado entre mortalidade e recrutamento resultaria apenas em flutuações florísticoestruturais.

O objetivo deste trabalho foi analisar a dinâmica da comunidade arbórea na mata de galeria do Gama, referente a um período de 19 anos.

\section{Material e métodos}

Área de estudo - Este estudo foi realizado em uma área de 64 ha da mata de galeria do Gama, localizada na Fazenda

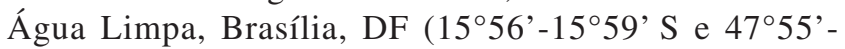
$47^{\circ} 58^{\prime} \mathrm{W}$ ), a uma altitude média de $1100 \mathrm{~m}$.

O clima da região, segundo o sistema de classificação de Köppen, é do tipo Aw, com verão chuvoso e inverno seco (Nimer 1989). A umidade relativa do ar entre os meses de Maio a Setembro está abaixo de 70\%; a umidade mínima ocorre em Agosto, com uma média de 47\%; as médias anuais de temperatura e precipitação são $22,2{ }^{\circ} \mathrm{C}$ e $1281 \mathrm{~mm}$, respectivamente, de acordo com os dados da Estação Climatológica da Reserva Ecológica do IBGE para o período de 1985 a 2004.

A mata de galeria do Gama ocorre em solo distrófico e com elevada saturação de alumínio, em área plana e bem drenada (Felfili 1994). Apresenta elevada riqueza com 93 espécies arbóreas com poucas espécies concentrando grande parte da densidade de indivíduos. Apenas 12 espécies apresentaram mais de 20 indivíduos ha $^{-1} \mathrm{e}$ as demais ocorreram em baixa densidade, dentre as quais 21 apresentaram menos que um indivíduos ha-1. A área basal foi de $30 \mathrm{~m}^{2} \mathrm{ha}^{-1}$, a altura do dossel esteve em torno de $20 \mathrm{~m}$ e o diâmetro variou de 30 a $95 \mathrm{~cm}$ (Felfili 1994).

Inventário contínuo - $\mathrm{O}$ estudo teve como base os dados provenientes do inventário contínuo da mata de galeria do Gama, obtidos em 151 parcelas permanentes $(10 \times 20 \mathrm{~m})$ alocadas sistematicamente ao longo de 10 transectos dispostos a cada $100 \mathrm{~m}$ perpendicularmente ao Ribeirão Gama. O comprimento de cada transecto variou de acordo com a largura da mata (Felfili 1994).

Todos os indivíduos com cerca de $10 \mathrm{~cm}$ de diâmetro à altura do peito (DAP) foram incluídos na amostragem. Estes foram mapeados nas parcelas em um sistema de coordenadas x/y marcados e mensurados em 1985, 1988, 1991, 1994, 1995 e 1997), 1994 e 1999 (Salgado 2003). Para esse estudo foi realizado inventário da mata em 2004 segundo os métodos usados anteriormente, avaliando as mesmas variáveis mensuradas nos inventários anteriores conforme descrito por Felfili (1995).

As circunferências à altura do peito (CAPs) maiores ou iguais a $31 \mathrm{~cm}$ de todos os troncos foram medidas com fita de costureira e posteriormente transformadas em diâmetro.

O recrutamento foi considerado para aqueles indivíduos jovens que alcançaram o tamanho mínimo de amostragem (DAP $\geq 10 \mathrm{~cm}$ ) durante o período estudado. A mortalidade foi considerada como o número de árvores mortas, incluindo aquelas encontradas em pé, aquelas caídas no chão e aquelas ausentes na remedição.

O conjunto de dados proveniente dos inventários realizados foi utilizado para os cálculos das taxas demográficas para os seis períodos de amostragem (1985 a 1988, 1988 a 1991, 1991 a 1994, 1994 a 1999, 1999 a 2004 e 1985 a 2004).

Parâmetros da dinâmica - Os dados obtidos nos seis períodos de amostragem foram utilizados para estimar o incremento periódico anual (IPA) em diâmetro, expresso em $\mathrm{cm} \mathrm{ano}^{-1}$, calculado por meio da diferença do diâmetro entre a segunda e primeira medições dividida pelo tempo transcorrido em anos, de acordo com a fórmula descrita por Finger (1992). Em cada período, foram determinados a média do IPA e o coeficiente de variação dos incrementos para toda a comunidade. Para as espécies que apresentaram, no mínimo, 30 indivíduos vivos no período 1985 a 1988 foi calculado o valor médio do IPA em todos os períodos. Foram selecionadas as espécies com pelo menos 30 indivíduos no primeiro período comparado para manter a mesma série de dados na análise temporal. 
Para cada classe de diâmetro da comunidade, foram calculados os incrementos periódicos anual (IPA) para todos os períodos estudados.

Para os cálculos do IPA, os incrementos negativos registrados para alguns indivíduos foram transformados para o valor zero, uma vez que se observou que essas árvores apresentaram algum tipo de anormalidades devido à perda de casca e/ou outras irregularidades, considerando-se, portanto, que não houve crescimento no período, conforme adotado por Felfili (1995) e que os incrementos negativos foram menos do que $0,01 \%$ da totalidade dos incrementos analisados.

A taxa de mortalidade média anual e o recrutamento foram calculados para a comunidade arbórea amostrada em cada período e por classe de diâmetro. Os intervalos de classe de diâmetro sendo os mesmos adotados em estudos anteriores por Fefili 1995 com base em fórmula sugerida por Spiegel.

A distribuição em classes mostra que comunidades tropicais apresentam um padrão de J-reverso, onde a maioria dos indivíduos e de espécies ocorrem nas menores classes onde grande parte dos processos diâmicos ocorrem de forma mais intensa como a mortalidade e recrutamento. Algumas espécies de menor porte, são, inclusive, restritas nas menores classes, levando à necessidade da análise das taxas de dinâmica por classe de diâmetro.

Para cada classe de diâmetro da comunidade, foi calculada a taxa de mortalidade para o período de 1985 a 2004. Para o cálculo das taxas, utilizou-se o modelo logarítmico, seguindo as recomendações de Swaine \& Lieberman (1987), o qual assume que a mortalidade declina logaritmicamente com o tempo, de acordo com a equação:

$$
m(r)=\left(100 \cdot\left(\ln \left(n_{0}\right)-\ln \left(n_{\mathrm{i}}\right)\right)\right) / t,
$$

onde $m$ é a taxa de mortalidade média anual $(m>0) ; r$ é a taxa de recrutamento médio anual $(r<0) ; n_{0}$ é o número de indivíduos na primeira medição; $n_{i}$ é o número de indivíduos sobreviventes na segunda medição (no caso de recrutamento, é o número de sobreviventes somado ao número de recrutas) e $t$ é o número de anos entre as duas medições.

A partir das taxas de mortalidade e de recrutamento, foram determinados os respectivos tempos de meia-vida $\left(\mathrm{t}_{1 / 2}\right)$ (Swaine \& Lieberman 1987) e de duplicação $\left(t_{2}\right)$ (Korning \& Balslev 1994a).

O tempo de meia vida $\left(t_{1 / 2}\right)$ representa o tempo necessário para a população ou comunidade se reduzir em tamanho à metade a partir da presente taxa de mortalidade, sendo que, quanto maior a taxa de mortalidade, menor será $\mathrm{t}_{1 / 2}$ (Swaine \& Lieberman 1987). O tempo de duplicação $\left(\mathrm{t}_{2}\right)$ é o tempo para duplicar o tamanho de uma população ou comunidade a partir da presente taxa de recrutamento e quanto maior o recrutamento menor o $t_{2}$ (Korning \& Balslev 1994a).

De acordo com o proposto por Korning \& Balslev (1994a), foram calculados os valores de estabilidade e de rotatividade, expressos em anos. A estabilidade foi calculada a partir da diferença entre o tempo de duplicação e de meia vida; quanto mais próximo de zero mais estável é a comunidade. A rotatividade é a média aritmética entre a meia vida e o tempo de duplicação e quanto menor o valor mais dinâmica é a comunidade.

A média anual da taxa de rotatividade, expressa em $\%$ ano $^{-1}$, foi calculada a partir da média entre as taxas de recrutamento e de mortalidade (Korning \& Balslev 1994a). Neste caso, quanto maior a taxa de rotatividade, maiores as taxas de mortalidade e/ou de recrutamento e menor a rotatividade.

Os incrementos periódicos anual (IPA) médios dos períodos de 1985 a 1988, 1988 a 1991, 1991 a 1994, 1994 a 1999, 1999 a 2004 e 1985 a 2004 foram comparados entre si pelo teste de $\chi^{2}$ no nível de significância de $5 \%$ (Zar 1999), para verificar a existência ou não de diferenças entre as médias dos períodos estudados.

Foi calculada a correlação linear de Pearson entre mortalidade $\times$ densidade, mortalidade $\times$ área basal, recrutamento $\times$ densidade e recrutamento $\times$ área basal. Para os cálculos foi usado o software SYSTAT 7.0.

\section{Resultados}

A listagem das espécies arbóreas amostradas na mata de galeria do Gama durante o intervalo de 1985 a 2004 encontra-se na tabela 1 . Verificou-se que $83 \%$ do total de espécies registrado na mata ocorreram em todas as ocasiões durante o período de 19 anos de estudo, não sendo observado padrão de entrada de espécies a partir do segundo levantamento, encontrando-se novas espécies em todos os anos posteriores a 1985 (tabela 1). Entre 1985 e 2004, 14 novas espécies ingressaram na área enquanto apenas seis espécies presentes em 1985 dela desapareceram até 2004. Apenas duas espécies não registradas em 1985 surgiram no decorrer do tempo mas desapareceram antes de 2004.

Incremento - O incremento periódico anual (IPA) da comunidade no período de 19 anos (1985 a 2004) foi de $0,22 \mathrm{~cm}$ ano $^{-1}$. Foram registrados valores médios similares em todos os períodos analisados, variando de 0,21 a $0,26 \mathrm{~cm}^{2} \mathrm{ano}^{-1}$. A variação entre os incrementos calculados em cada período foi elevada, variando de $84,28 \%$ no período de 1985 a 2004 a 142,98\% no período de 1988 a 1991 (tabela 2).

O incremento periódico anual (IPA) da comunidade arbórea para todos os períodos mostrou uma tendência crescente da menor classe de diâmetro para as subsequentes até $52 \mathrm{~cm}$ de DAP, onde estão concentrados cerca de $95 \%$ dos indivíduos. A partir desse diâmetro, as classes diamétricas possuem poucos indivíduos, observando-se uma grande oscilação nas médias do incremento (figura 1). 
Tabela 1. Lista das espécies lenhosas registradas na mata de galeria do Gama, Fazenda Água Limpa, Brasília, DF. As espécies estão em ordem alfabética das famílias botânicas (108 espécies e 48 famílias), seguidas de suas respectivas ocorrências durante o intervalo de 1985 a 2004.

Table 1. List of the woody species that occur at the Gama gallery forest, Água Limpa farm, Brasília, Brazil. The species are in alphabetic order of the botanical families (108 species and 48 families) followed by their respective occurrence between 1985 and 2004.

\begin{tabular}{|c|c|c|c|c|c|c|}
\hline Família / Espécie* & 1985 & 1988 & 1991 & 1994 & 1999 & 2004 \\
\hline \multicolumn{7}{|l|}{ ANACARDIACEAE } \\
\hline Astronium fraxinifolium Schott. ex Spreng & $\mathrm{x}$ & $\mathrm{x}$ & $\mathrm{x}$ & $\mathrm{x}$ & $\mathrm{x}$ & $\mathrm{x}$ \\
\hline Tapirira guianensis Aubl. & $\mathrm{x}$ & $\mathrm{x}$ & $\mathrm{x}$ & $\mathrm{x}$ & $\mathrm{x}$ & $\mathrm{x}$ \\
\hline \multicolumn{7}{|l|}{ ANNONACEAE } \\
\hline Cardiopetalum calophyllum Schltdl. & & & & $\mathrm{x}$ & & \\
\hline Guatteria sellowiana Schltdl. & $\mathrm{x}$ & $\mathrm{x}$ & $\mathrm{x}$ & $\mathrm{x}$ & $\mathrm{x}$ & $\mathrm{x}$ \\
\hline Xylopia emarginata Mart. & $\mathrm{x}$ & $\mathrm{x}$ & $\mathrm{x}$ & $\mathrm{x}$ & $\mathrm{x}$ & $\mathrm{x}$ \\
\hline Xylopia sericea A. St.-Hil. & $\mathrm{x}$ & $\mathrm{x}$ & $\mathrm{x}$ & $\mathrm{x}$ & $\mathrm{x}$ & $\mathrm{x}$ \\
\hline \multicolumn{7}{|l|}{ APOCYNACEAE } \\
\hline Aspidosperma cylindrocarpon Müll. Arg. & $\mathrm{x}$ & $\mathrm{x}$ & $\mathrm{x}$ & $\mathrm{x}$ & $\mathrm{x}$ & $\mathrm{x}$ \\
\hline Aspidosperma discolor A. DC. & $\mathrm{x}$ & $\mathrm{x}$ & $\mathrm{x}$ & $\mathrm{x}$ & $\mathrm{x}$ & $\mathrm{x}$ \\
\hline Aspidosperma parvifolium A. DC. & $\mathrm{x}$ & $\mathrm{x}$ & $\mathrm{x}$ & $\mathrm{x}$ & $\mathrm{x}$ & $\mathrm{x}$ \\
\hline Aspidosperma sp. & $\mathrm{x}$ & $\mathrm{x}$ & $\mathrm{x}$ & $\mathrm{x}$ & $\mathrm{x}$ & $\mathrm{x}$ \\
\hline Aspidosperma subincanum Mart. & $\mathrm{x}$ & $\mathrm{x}$ & $\mathrm{x}$ & $\mathrm{x}$ & $\mathrm{x}$ & $\mathrm{x}$ \\
\hline \multicolumn{7}{|l|}{ AQUIFOLIACEAE } \\
\hline Ilex sp. & & & & & $\mathrm{x}$ & $\mathrm{x}$ \\
\hline \multicolumn{7}{|l|}{ ARALIACEAE } \\
\hline Gilibertia cuneata March. & $\mathrm{x}$ & $\mathrm{x}$ & $\mathrm{x}$ & $\mathrm{x}$ & $\mathrm{x}$ & $\mathrm{x}$ \\
\hline Schefflera morototonii (Aubl.) DC. Frodin & $\mathrm{x}$ & $\mathrm{x}$ & $\mathrm{x}$ & $\mathrm{x}$ & $\mathrm{x}$ & $\mathrm{x}$ \\
\hline \multicolumn{7}{|l|}{ ASTERACEAE } \\
\hline Piptocarpha macropoda (DC.) Baker & $\mathrm{x}$ & $\mathrm{x}$ & $\mathrm{x}$ & $\mathrm{x}$ & $\mathrm{x}$ & $\mathrm{x}$ \\
\hline \multicolumn{7}{|l|}{ BIGNONIACEAE } \\
\hline Jacaranda caroba (Vell.) A. DC. & & & & $\mathrm{x}$ & $\mathrm{x}$ & $\mathrm{x}$ \\
\hline \multicolumn{7}{|l|}{ BOMBACACEAE } \\
\hline Eriotheca gracilipes (K. Schum.) A. Robyns & $\mathrm{x}$ & $\mathrm{x}$ & $\mathrm{x}$ & $\mathrm{x}$ & $\mathrm{x}$ & $\mathrm{x}$ \\
\hline \multicolumn{7}{|l|}{ BORAGINACEAE } \\
\hline Cordia sellowiana Cham. & $\mathrm{x}$ & $\mathrm{x}$ & $\mathrm{x}$ & $\mathrm{x}$ & $\mathrm{x}$ & $\mathrm{x}$ \\
\hline \multicolumn{7}{|l|}{ BURSERACEAE } \\
\hline Protium heptaphyllum (Aubl) Marchand & $\mathrm{x}$ & $\mathrm{x}$ & $\mathrm{x}$ & $\mathrm{x}$ & $\mathrm{x}$ & $\mathrm{x}$ \\
\hline \multicolumn{7}{|l|}{ CECROPIACEAE } \\
\hline Cecropia pachystachia Trécul & $\mathrm{x}$ & $\mathrm{x}$ & $\mathrm{x}$ & $\mathrm{x}$ & $\mathrm{x}$ & $\mathrm{x}$ \\
\hline \multicolumn{7}{|l|}{ CELASTRACEAE } \\
\hline Maytenus alaternoides Reissek & $\mathrm{x}$ & $\mathrm{x}$ & $\mathrm{x}$ & $\mathrm{x}$ & $\mathrm{x}$ & $\mathrm{x}$ \\
\hline \multicolumn{7}{|l|}{ CHRYSOBALANACEAE } \\
\hline Hirtella glandulosa Spreng. & $\mathrm{x}$ & $\mathrm{x}$ & $\mathrm{x}$ & $\mathrm{x}$ & $\mathrm{x}$ & $\mathrm{x}$ \\
\hline Licania apetala (E. Mey.) Fritsch & $\mathrm{x}$ & $\mathrm{x}$ & $\mathrm{x}$ & $\mathrm{x}$ & $\mathrm{x}$ & $\mathrm{x}$ \\
\hline \multicolumn{7}{|l|}{ CLUSIACEAE } \\
\hline Calophyllum brasiliense Cambess. & $\mathrm{x}$ & $\mathrm{x}$ & $\mathrm{x}$ & $\mathrm{x}$ & $\mathrm{x}$ & $\mathrm{x}$ \\
\hline Clusia sp. & & & & & $\mathrm{x}$ & \\
\hline Rheedia brasiliensis (Mart.) Planc. \& Triana & & & & & & $\mathrm{x}$ \\
\hline Vismia sp. & $\mathrm{x}$ & $\mathrm{x}$ & $\mathrm{x}$ & $\mathrm{x}$ & $\mathrm{x}$ & $\mathrm{x}$ \\
\hline \multicolumn{7}{|l|}{ COMBRETACEAE } \\
\hline Terminalia argentea (Camb.) Mart. & $\mathrm{x}$ & $\mathrm{x}$ & $\mathrm{x}$ & $\mathrm{x}$ & $\mathrm{x}$ & $\mathrm{x}$ \\
\hline Terminalia brasiliensis (Cambess. ex A. St.-Hil.) & $\mathrm{x}$ & $\mathrm{x}$ & $\mathrm{x}$ & $\mathrm{x}$ & $\mathrm{x}$ & $\mathrm{x}$ \\
\hline \multicolumn{7}{|l|}{ CUNNONIACEAE } \\
\hline Lamanonia ternata Vell. & $\mathrm{x}$ & $\mathrm{x}$ & $\mathrm{x}$ & $\mathrm{x}$ & $\mathrm{x}$ & $\begin{array}{c}\mathrm{x} \\
\text { intinuc }\end{array}$ \\
\hline
\end{tabular}


continuação

\begin{tabular}{|c|c|c|c|c|c|c|}
\hline Família / Espécie* & 1985 & 1988 & 1991 & 1994 & 1999 & 2004 \\
\hline \multicolumn{7}{|l|}{ DICHAPETALACEAE } \\
\hline $\begin{array}{l}\text { Tapura amazonica Poepp. } \\
\text { EBENACEAE }\end{array}$ & $\mathrm{x}$ & $\mathrm{x}$ & $\mathrm{x}$ & $\mathrm{x}$ & $\mathrm{x}$ & $\mathrm{x}$ \\
\hline \multicolumn{7}{|l|}{ ELAEOCARPACEAE } \\
\hline \multicolumn{6}{|l|}{ ERYTHROXYLACEAE } & $\mathrm{x}$ \\
\hline \multicolumn{7}{|l|}{ EUPHORBIACEAE } \\
\hline Alchornea irucurana Casar. & $\mathrm{x}$ & $\mathrm{X}$ & $\mathrm{x}$ & $\mathrm{X}$ & $\mathrm{x}$ & $\mathrm{x}$ \\
\hline Maprounea guianensis Aubl. & $\mathrm{x}$ & $\mathrm{X}$ & $\mathrm{x}$ & $\mathrm{X}$ & $\mathrm{x}$ & $\mathrm{x}$ \\
\hline $\begin{array}{l}\text { Pera glabrata (Schott) Poepp. ex Baill. } \\
\text { Richeria grandis Vahl. }\end{array}$ & $\mathrm{x}$ & $\mathrm{x}$ & $\mathrm{x}$ & $\mathrm{x}$ & $\mathrm{x}$ & $\begin{array}{l}\mathrm{X} \\
\mathrm{X}\end{array}$ \\
\hline \multicolumn{7}{|l|}{ FLACOURTIACEAE } \\
\hline \multicolumn{6}{|l|}{ HIPPOCRATEACEAE } & $\mathrm{X}$ \\
\hline Cheiloclinium cognatum (Miers) A. C. Sm. & $\mathrm{x}$ & $\mathrm{X}$ & $\mathrm{x}$ & $\mathrm{x}$ & $\mathrm{x}$ & $\mathrm{x}$ \\
\hline \multicolumn{7}{|l|}{ HUMIRIACEAE } \\
\hline \multicolumn{7}{|l|}{ ICACINACEAE } \\
\hline Emmotum nitens (Benth.) Miers & $\mathrm{x}$ & $\mathrm{x}$ & $\mathrm{x}$ & $\mathrm{x}$ & $\mathrm{x}$ & $\mathrm{x}$ \\
\hline \multicolumn{7}{|l|}{ LAURACEAE } \\
\hline Aniba heringerii Vattimo & $\mathrm{x}$ & $\mathrm{x}$ & $\mathrm{x}$ & $\mathrm{X}$ & $\mathrm{x}$ & $\mathrm{x}$ \\
\hline Cryptocaria aschersoniana $\mathrm{Mez}$ & $\mathrm{x}$ & $\mathrm{x}$ & $\mathrm{x}$ & $\mathrm{x}$ & $\mathrm{x}$ & $\mathrm{x}$ \\
\hline Nectandra mollis (Kunth) Nees & $\mathrm{x}$ & $\mathrm{X}$ & $\mathrm{x}$ & $\mathrm{x}$ & $\mathrm{x}$ & $\mathrm{x}$ \\
\hline Ocotea spixiana (Nees) Mez & $\mathrm{x}$ & $\mathrm{x}$ & $\mathrm{x}$ & $\mathrm{X}$ & $\mathrm{x}$ & $\mathrm{X}$ \\
\hline Persea fusca Mez & $\mathrm{x}$ & $\mathrm{x}$ & $\mathrm{x}$ & $\mathrm{X}$ & $\mathrm{x}$ & $\mathrm{x}$ \\
\hline \multicolumn{7}{|l|}{ LECYTHIDACEAE } \\
\hline Cariniana estrellensis (Raddi) Kuntze & $\mathrm{x}$ & $\mathrm{x}$ & $\mathrm{x}$ & $\mathrm{x}$ & $\mathrm{x}$ & $\mathrm{x}$ \\
\hline \multicolumn{7}{|l|}{ LEGUMINOSAE CAESALPINOIDEAE } \\
\hline Apuleia molaris Spruce ex Benth. & $\mathrm{x}$ & $\mathrm{x}$ & $\mathrm{x}$ & $\mathrm{X}$ & $\mathrm{x}$ & $\mathrm{x}$ \\
\hline Bauhinia rufa (Bong.) Steud. & $\mathrm{x}$ & $\mathrm{X}$ & $\mathrm{x}$ & $\mathrm{X}$ & $\mathrm{x}$ & $\mathrm{x}$ \\
\hline Copaifera langsdorffii Desf. & $\mathrm{x}$ & $\mathrm{x}$ & $\mathrm{x}$ & $\mathrm{X}$ & $\mathrm{x}$ & $\mathrm{x}$ \\
\hline Hymenaea stigonocarpa Mart. ex Hayne & $\mathrm{x}$ & $\mathrm{x}$ & $\mathrm{x}$ & $\mathrm{X}$ & $\mathrm{x}$ & $\mathrm{x}$ \\
\hline Platypodium elegans Vogel & $\mathrm{x}$ & $\mathrm{X}$ & $\mathrm{x}$ & $\mathrm{X}$ & $\mathrm{x}$ & $\mathrm{x}$ \\
\hline $\begin{array}{l}\text { Sclerolobium paniculatum Vogel var. paniculatum } \\
\text { LEGUMINOSAE MIMOSOIDEAE }\end{array}$ & $\mathrm{x}$ & $\mathrm{x}$ & $\mathrm{x}$ & $\mathrm{x}$ & $\mathrm{x}$ & $\mathrm{x}$ \\
\hline Inga alba (Sw.) Willd. & $\mathrm{x}$ & $\mathrm{x}$ & $\mathrm{x}$ & $\mathrm{X}$ & $\mathrm{x}$ & $\mathrm{x}$ \\
\hline \multicolumn{7}{|l|}{ LEGUMINOSAE PAPILIONOIDEAE } \\
\hline Acosmium subelegans (Mohlenbr.) Yakovlev & $\mathrm{x}$ & $\mathrm{x}$ & $\mathrm{x}$ & $\mathrm{x}$ & $\mathrm{x}$ & $\mathrm{x}$ \\
\hline Andira paniculata Benth. & $\mathrm{x}$ & $\mathrm{X}$ & $\mathrm{x}$ & $\mathrm{X}$ & $\mathrm{x}$ & $\mathrm{x}$ \\
\hline Machaerium acutifolium Vogel & $\mathrm{x}$ & $\mathrm{x}$ & $\mathrm{x}$ & $\mathrm{x}$ & $\mathrm{x}$ & $\mathrm{x}$ \\
\hline \multicolumn{7}{|l|}{ MALPIGHIACEAE } \\
\hline \multicolumn{6}{|l|}{ MELASTOMATACEAE } & $\mathrm{x}$ \\
\hline Miconia chartacea Triana & & & & & & $\mathrm{x}$ \\
\hline Miconia cuspidata Mart. ex Naudin & & & $\mathrm{x}$ & $\mathrm{X}$ & $\mathrm{x}$ & $\mathrm{x}$ \\
\hline Miconia dodecandra (Desv.) Cogn. & & & & & $\mathrm{x}$ & $\mathrm{x}$ \\
\hline Miconia punctata (Desr.) D. Don ex DC. & $\mathrm{x}$ & $\mathrm{x}$ & $\mathrm{x}$ & $\mathrm{x}$ & & \\
\hline Miconia sellowiana Naudin & $\mathrm{x}$ & $\mathrm{x}$ & $\mathrm{x}$ & $\mathrm{x}$ & $\mathrm{x}$ & $\mathrm{x}$ \\
\hline Mouriri graveolens Spruce \& Triana & & & & & $\mathrm{x}$ & $\mathrm{X}$ \\
\hline
\end{tabular}


continuação

Família / Espécie*

1985

1988

1991

1994

1999

2004

MELIACEAE

Cabralea canjerana (Vell.) Mart.

Trichilia catigua Adr. Juss

$\mathrm{X}$

MORACEAE

Pseudolmedia laevigata Trécul

Sorocea ilicifolia Miq.

MYRISTICACEAE

Virola sebifera Aubl.

MYRSINACEAE

Myrsine coriacea (Sw.) R. Br. ex Roem. \& Schult

Rapanea guianensis Aubl.

MYRTACEAE

Campomanesia velutina (Cambess.) O. Berg

Gomidesia lindeniana O. Berg.

Myrcia deflexa DC.

Myrcia sellowiana $\mathrm{O}$. Berg

Myrcia sp.

Siphoneugena densiflora O. Berg

Marlierea sp.

NYCTAGINACEAE

Guapira graciliflora (Schmidt) Lundell

OCHNACEAE

Ouratea castaneifolia (DC.) Engl.

OPILIACEAE

Agonandra brasiliensis Benth. \& Hook. f.

PROTEACEAE

Euplassa inaequalis (Pohl) Engl.

Roupala montana (Aubl.)

RUBIACEAE

Alibertia macrophylla K. Schum.

Amaioua guianensis Aubl.

Coussarea hydrangeifolia (Benth.) Müll.Arg.

Faramea sp.

Ferdinandusa speciosa Pohl

Guettarda viburnoides Cham \& Schltdl.

Ixora warmingii Müll. Arg.

Psychotria sp.

RUTACEAE

Metrodorea pubescens A. St.-Hil. \& Tul.

Zanthoxylum rhoifolium Lam.

SAPINDACEAE

Cupania vernalis Cambess.

Matayba guianensis Aubl.

SAPOTACEAE

Chrysophyllum marginatum (Hook. \& Arn.) Radlk

Micropholis venulosa (Mart. \& Eichler) Pierre

Pouteria ramiflora (Mart.) Radlk

SOLANACEAE

Solanum guianense Dunal

STYRACACEAE

Styrax camporum Pohl

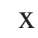

X

$\mathrm{X}$

$\mathrm{X}$

$\mathrm{X}$

X

$\mathrm{X}$

$\mathrm{X}$

$\mathrm{X}$

X
$\mathrm{X}$
$\mathrm{X}$

$\mathrm{X}$

$\mathrm{X}$

X

\title{
X
}

$\mathrm{X}$
$\mathrm{X}$

\author{
$\mathrm{X}$
$\mathrm{X}$
}

$\mathrm{X}$
$\mathrm{X}$

X

$\mathrm{X}$

$\mathrm{X}$
$\mathrm{X}$

X

X

$\mathrm{X}$
$\mathrm{X}$

$\begin{array}{ll}\mathrm{X} & \mathrm{X} \\ \mathrm{X} & \mathrm{X}\end{array}$

X

X

$x$

X

X $\quad$ X

$\begin{array}{ll}\mathrm{X} & \mathrm{X} \\ \mathrm{X} & \mathrm{X}\end{array}$

$\begin{array}{lll}X & X & X \\ X & X & X\end{array}$

$\mathrm{X}$

X

$\mathrm{X}$
$\mathrm{X}$

$$
\mathrm{X}
$$

X

$\mathrm{X}$
$\mathrm{X}$
$\mathrm{X}$

X

X

$\mathrm{X}$
$\mathrm{X}$

X

$\mathrm{X}$
$\mathrm{X}$

$\mathrm{X}$
$\mathrm{X}$
$\mathrm{X}$
$\mathrm{X}$
$\mathrm{X}$
$\mathrm{X}$
$\mathrm{X}$

$\mathrm{X}$
$\mathrm{X}$

$\mathrm{X}$
$\mathrm{X}$

X

X

$\begin{array}{lll}X & X & X \\ X & X & X\end{array}$

\begin{tabular}{|c|c|c|c|}
\hline $\mathrm{X}$ & $X$ & $\mathrm{X}$ & $\mathrm{X}$ \\
\hline$X$ & $\mathrm{X}$ & $X$ & $\mathrm{X}$ \\
\hline & & $\mathrm{X}$ & $\mathrm{X}$ \\
\hline $\mathrm{X}$ & $\mathrm{X}$ & $\mathrm{X}$ & $\mathrm{X}$ \\
\hline$X$ & $X$ & & \\
\hline$X$ & $\mathrm{X}$ & $\mathrm{X}$ & $\mathrm{X}$ \\
\hline $\mathrm{X}$ & $\mathrm{X}$ & $X$ & $\mathrm{X}$ \\
\hline $\mathrm{X}$ & $\mathrm{X}$ & & \\
\hline $\mathrm{X}$ & $X$ & X & X \\
\hline $\mathrm{X}$ & $\mathrm{X}$ & X & X \\
\hline $\mathrm{X}$ & $\mathrm{X}$ & $\mathrm{X}$ & $\mathrm{X}$ \\
\hline$X$ & $X$ & $X$ & $\mathrm{X}$ \\
\hline$X$ & $\mathrm{X}$ & X & X \\
\hline $\mathrm{X}$ & $X$ & $\mathrm{X}$ & $\mathrm{X}$ \\
\hline $\mathrm{X}$ & $\mathrm{X}$ & $\mathrm{X}$ & $\mathrm{X}$ \\
\hline $\mathrm{X}$ & $\mathrm{X}$ & X & X \\
\hline $\mathrm{X}$ & $\mathrm{X}$ & $\mathrm{X}$ & $\mathrm{X}$ \\
\hline
\end{tabular}


continuação

\begin{tabular}{|c|c|c|c|c|c|c|}
\hline Família / Espécie* & 1985 & 1988 & 1991 & 1994 & 1999 & 2004 \\
\hline \multicolumn{7}{|l|}{ TILIACEAE } \\
\hline $\begin{array}{l}\text { Luehea paniculata Mart. \& Zucc. } \\
\text { VERBENACEAE }\end{array}$ & $\mathrm{x}$ & $\mathrm{x}$ & $\mathrm{x}$ & $\mathrm{x}$ & $\mathrm{x}$ & $\mathrm{x}$ \\
\hline $\begin{array}{l}\text { Aegiphila lhotzkiana Cham. } \\
\text { VOCHYSIACEAE }\end{array}$ & $\mathrm{x}$ & $\mathrm{x}$ & $\mathrm{x}$ & $\mathrm{x}$ & $\mathrm{x}$ & $\mathrm{x}$ \\
\hline Callisthene major Mart. & $\mathrm{x}$ & $\mathrm{x}$ & $\mathrm{x}$ & $\mathrm{x}$ & $\mathrm{x}$ & $\mathrm{x}$ \\
\hline Qualea dichotoma (Mart.) Warm. & $\mathrm{x}$ & $\mathrm{x}$ & $\mathrm{X}$ & $\mathrm{X}$ & $\mathrm{x}$ & $\mathrm{x}$ \\
\hline Qualea muiltiflora Mart. & $\mathrm{x}$ & $\mathrm{x}$ & $\mathrm{x}$ & $\mathrm{x}$ & $\mathrm{x}$ & $\mathrm{x}$ \\
\hline Vochysia tucanorum Mart. & $\mathrm{x}$ & $\mathrm{x}$ & $\mathrm{x}$ & $\mathrm{x}$ & $\mathrm{x}$ & $\mathrm{x}$ \\
\hline
\end{tabular}

* Não estão representadas na tabela três espécies identificadas até o nível de família: Lauraceae indet $(\mathrm{n}=2)$, Leguminosae indet $(\mathrm{n}=7)$ e Moraceae indet $(\mathrm{n}=2)$; todas registradas em todos os levantamentos.

* Three species identified only to Family are not in the table: Lauraceae indet $(\mathrm{n}=2)$, Leguminosae indet $(\mathrm{n}=7)$ and Moraceae indet $(\mathrm{n}=2)$, all present in all surveys.

Tabela 2. Incremento periódico anual (IPA) médio e coeficiente de variação (CV\%) para a comunidade lenhosa (DAP $\geq 10 \mathrm{~cm}$ ) da mata de galeria do Gama entre os anos de 1985 a 2004, Fazenda Água Limpa, Brasília, DF. ( $N=$ número de indivíduos).

Table 2. Average periodic annual increase (IPA) and variation coefficient (CV\%) of the woody community (DAP $\geq 10 \mathrm{~cm})$ of the Gama gallery forest between 1985 and 2004. Água Limpa farm, Brasília, Brazil. ( $N=$ number of individuals).

\begin{tabular}{lcccccc}
\hline & \multicolumn{4}{c}{ Períodos } \\
\cline { 2 - 7 } & $1985-88$ & $1988-91$ & $1991-94$ & $1994-99$ & $1999-04$ & $1985-04$ \\
\hline Média $(\mathrm{cm})$ & $0,26^{\mathrm{ns}}$ & $0,21^{\mathrm{ns}}$ & $0,26^{\mathrm{ns}}$ & $0,21^{\mathrm{ns}}$ & $0,24^{\mathrm{ns}}$ & $0,22^{\mathrm{ns}}$ \\
$\mathrm{CV}(\%)$ & 115 & 143 & 136 & 133 & 120 & 84 \\
$N$ & 1756 & 1770 & 1763 & 1554 & 1634 & 1132 \\
\hline
\end{tabular}

ns as médias não diferem a $5 \%$ de significância, pelo teste de $\chi^{2}(P>0,05)$.

ns the difference between the averages is not significant in the $\chi^{2}(P>0.05)$.

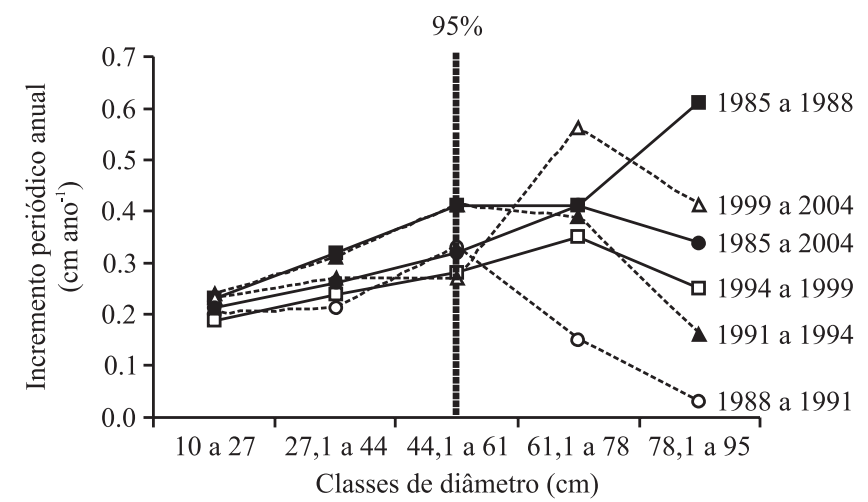

Figura 1. Incremento periódico anual (IPA), por classes de diâmetro, para a comunidade arbórea (DAP $\geq 10 \mathrm{~cm}$ ) da mata de galeria do Gama, entre os anos de 1985 a 2004, Fazenda Água Limpa, Brasília, DF. A linha tracejada vertical representa o diâmetro (DAP $=52 \mathrm{~cm}$ ) abaixo do qual se encontra cerca de $95 \%$ dos indivíduos arbóreos amostrados.

Figure 1. Periodic annual increase (IPA), by diameter classes, of the woody community (DAP $\geq 10 \mathrm{~cm}$ ) of the Gama gallery forest between 1985 and 2004. Água Limpa farm, Brasília, Brazil. The dotted line indicates the diameter (DAP $=52 \mathrm{~cm})$ that separates $95 \%$ of the sampled individuals.
O incremento periódico anual das espécies que apresentaram, no mínimo, 30 indivíduos vivos entre 1985 a 1988, por período, encontra-se na tabela 3. Das 97 espécies vivas entre 1985 a 1988, apenas 18 apresentaram 30 ou mais indivíduos. As espécies Cryptocaria aschersoniana, Piptocarpha macropoda, Protium heptaphyllum e Tapirira guianensis apresentaram incremento médio em diâmetro superior ao da comunidade em todos os períodos analisados. O incremento das espécies Cheiloclinium cognatum, Guatteria sellowiana, Lamanonia ternata, Licania apetala, Matayba guianensis e Ouratea castaneifolia sempre estiveram abaixo dos respectivos incrementos calculados para a comunidade nos seis períodos. O maior valor absoluto de incremento registrado foi $0,51 \mathrm{~cm}^{2} \mathrm{ano}^{-1}$ para Tapirira guianensis, uma espécie emergente não pioneira (Felfili 1997c) cujo diâmetro máximo alcançado na área foi de $64 \mathrm{~cm}$.

Mortalidade e recrutamento - Durante o intervalo de 19 anos (1985 a 2004), a mortalidade total foi de $42 \%$, o que resultou numa taxa de $2,87 \%$ ano $^{-1}$, baseado no modelo logarítmico. As taxas médias anuais de 
Tabela 3. Incremento periódico anual de diâmetro (IPA) para as espécies com, no mínimo, 30 indivíduos vivos no período 1985 a 1988, na comunidade lenhosa (DAP $\geq 10 \mathrm{~cm}$ ) da mata de galeria do Gama, Fazenda Água Limpa, Brasília, DF. As espécies estão listadas em ordem decrescente do número de indivíduos sobreviventes no período.

Table 3. Annual periodic increase in diameter (IPA) of species with at least 30 individuals alive between 1985 and 2004 in the woody community (DAP $\geq 10 \mathrm{~cm}$ ) of the Gama gallery forest. Água Limpa farm, Brasília, Brazil. The species are listed in descending order of number of individuals alive in the period.

\begin{tabular}{lccccccc}
\hline \multirow{2}{*}{ Espécie } & \multicolumn{7}{c}{ IPA $\left(\mathrm{cm} \mathrm{ano}^{-1}\right)$} \\
\cline { 2 - 7 } & $N$ & $1985-88$ & $1988-91$ & $1991-94$ & $1994-99$ & $1999-04$ & $1985-04$ \\
\hline Metrodorea pubescens & 107 & $0,27^{*}$ & $0,21^{*}$ & 0,25 & $0,24^{*}$ & $0,24^{*}$ & $0,24^{*}$ \\
Licania apetala & 96 & 0,18 & 0,14 & 0,20 & 0,14 & 0,20 & 0,17 \\
Guatteria sellowiana & 96 & 0,14 & 0,19 & 0,20 & 0,12 & 0,17 & 0,16 \\
Amaioua guianensis & 96 & 0,18 & $0,22^{*}$ & 0,22 & 0,14 & 0,16 & 0,15 \\
Cheiloclinium cognatum & 96 & 0,14 & 0,15 & 0,17 & 0,14 & 0,11 & 0,13 \\
Lamanonia ternata & 86 & 0,23 & 0,17 & 0,25 & 0,14 & 0,19 & 0,20 \\
Aspidosperma parviflorum & 83 & $0,31^{*}$ & 0,13 & $0,26^{*}$ & $0,22^{*}$ & 0,21 & $0,23^{*}$ \\
Protium heptaphyllum & 76 & $0,34^{*}$ & $0,22^{*}$ & $0,36^{*}$ & $0,22^{*}$ & $0,34^{*}$ & $0,29^{*}$ \\
Copaifera langsdorffii & 74 & $0,30^{*}$ & 0,20 & 0,25 & $0,21^{*}$ & $0,30^{*}$ & $0,26^{*}$ \\
Nectandra mollis & 69 & $0,29^{*}$ & 0,20 & 0,24 & $0,26^{*}$ & 0,23 & $0,25^{*}$ \\
Tapirira guianensis & 63 & $0,41^{*}$ & $0,38^{*}$ & $0,54^{*}$ & $0,41^{*}$ & $0,47^{*}$ & $0,43^{*}$ \\
Piptocarpha macropoda & 54 & $0,36^{*}$ & $0,51^{*}$ & $0,31^{*}$ & $0,45^{*}$ & $0,41^{*}$ & $0,45^{*}$ \\
Cryptocaria aschersoniana & 45 & $0,33^{*}$ & $0,31^{*}$ & $0,31^{*}$ & $0,27^{*}$ & $0,27^{*}$ & $0,28^{*}$ \\
Callisthene major & 39 & $0,30^{*}$ & $0,22^{*}$ & $0,44^{*}$ & $0,29^{*}$ & 0,22 & $0,28^{*}$ \\
Ouratea castaneifolia & 37 & 0,19 & 0,13 & 0,16 & 0,16 & 0,10 & 0,12 \\
Maytenus alaternoides & 36 & 0,15 & 0,19 & 0,13 & $0,28^{*}$ & 0,19 & 0,20 \\
Matayba guianensis & 31 & 0,20 & 0,19 & 0,18 & 0,16 & 0,13 & 0,18 \\
Emmotum nitens & 30 & 0,32 & 0,20 & 0,18 & 0,19 & 0,15 & 0,21 \\
\hline
\end{tabular}

* Médias de incremento de diâmetro das espécies acima da média da comunidade para os períodos de 1985 a 1988 e 1991 a 1994 (IPA $\geq 0,26 \mathrm{~cm}$ ano-1), 1988 a 1991 e 1994 a 1999 (IPA $\left.\geq 0,21 \mathrm{~cm} \mathrm{ano}^{-1}\right), 1999$ a 2004 (IPA $\geq 0,24 \mathrm{~cm}_{\text {ano }}^{-1}$ ) e 1985 a 2004 (IPA $\geq 0,22 \mathrm{~cm}$ ano $\left.{ }^{-1}\right),(N=$ número de indivíduos vivos em 1985 ). * Average diameter increase of the species above the average in the comunity in the period of 1985 to 1998 and 1991 to 1994 (IPA $\geq 0.26 \mathrm{~cm}^{-1}$ year-1), 1988 to 1991 and 1994 to 1999 (IPA $\geq 0.21 \mathrm{~cm}$ year-1 $\left.^{-1}\right), 1999$ to 2004 (IPA $\geq 0.24 \mathrm{~cm} \mathrm{year}^{-1}$ ) and 1985 to 2004 (IPA $\geq 0.22 \mathrm{~cm}_{\text {year }}{ }^{-1}$ ). $(N=$ number of individuals alive in 1985).

mortalidade apresentaram menor variação para os três primeiros períodos (1985 a 1988, 1988 a 1991 e 1991 a 1994), quando comparados com os períodos sucessivos (1994 a 1999 e 1999 a 2004), os quais apresentaram a maior $\left(4,15 \%\right.$ ano $\left.^{-1}\right)$ e a menor $\left(1,64 \%\right.$ ano $\left.^{-1}\right)$ taxa de mortalidade, respectivamente (tabela 4 ).

Na figura 2 observa-se a mortalidade da comunidade, por classe de diâmetro, para o período de 1985 a 2004. O maior valor foi registrado para a primeira classe $\left(3,09 \%\right.$ ano $\left.^{-1}\right)$, que correspondeu a aproximadamente $82 \%$ de todos os indivíduos que morreram. As menores taxas de mortalidade foram registradas para as duas últimas classes de diâmetro $\left(1,63 \%\right.$ e 1,51\% ano $\left.{ }^{-1}\right)$.

Observou-se uma correlação positiva significativa tanto para mortalidade e densidade $(r=0,88 ; P<0,0001)$ quanto para área basal e mortalidade $(r=0,60 ; P<0,0001)$ das espécies $(N=96)$ no período de 1985 a 2004.

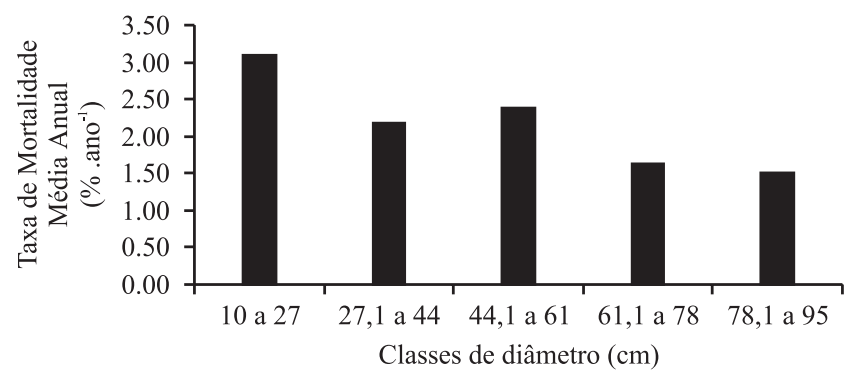

Figura 2. Taxa de mortalidade média anual, por classes de diâmetro, no período de 1985 a 2004, para a comunidade arbórea (DAP $\geq 10 \mathrm{~cm}$ ) na mata de galeria do Gama, Fazenda Água Limpa, Brasília, DF. (739 indivíduos mortos no período).

Figure 2. Average annual mortality rate, by diameter class, between 1985 and 2004 in the woody community (DAP $\geq$ $10 \mathrm{~cm}$ ) at the Gama gallery forest, Água Limpa farm, Brasília, Brazil. (739 deaths in the period). 
De acordo com o modelo logarítmico, o tempo de meia vida da comunidade arbórea da mata de galeria do Gama no período de 1985 a 2004 foi de 24,13 anos. Verificou-se que o tempo de meia-vida em quatro dos seis períodos comparados no intervalo de 1985 a 2004 variou de 19,46 a 24,13 anos. Valores discrepantes foram encontrados na comparação de 1994 a $1999\left(\mathrm{t}_{1 / 2}=42,16\right.$ anos) e de 1999 a 2004 ( $\mathrm{t}_{1 / 2}=16,72$ anos), onde foram encontradas as maiores e menores variações entre taxas de mortalidade e recrutamento, respectivamente (tabela 3 ).

Pode-se observar que os resultados obtidos para a comunidade arbórea estudada estão seguindo o padrão determinado pela meia vida no período de 1985 a 1988 (19,46 anos). Considerando o período de 19 anos de estudo
(1985-2004), observou-se que 42,07\% dos indivíduos amostrados em 1985 morreram até 2004.

No período de 1985 a 2004, o recrutamento total foi de $38 \%$. A taxa de recrutamento médio anual variou de $2,08 \%$, baseado no modelo logarítimo. A taxa de recrutamento anual variou de 2,8 a 3,07 ano $^{-1}$. Com exceção dos períodos de 1988-91 e 1999-04, as taxas de recrutamento calculadas para os períodos sucessivos sempre foram inferiores às taxas de mortalidade (tabela 4).

Observou-se uma correlação positiva significativa para o recrutamento e a densidade $(r=0,56 ; P<0,0001)$ das espécies $(N=104)$ no período de 1985 a 2004. Entretanto, a correlação entre a área basal e o recrutamento foi baixa $(r=0,25, P<0,05)$.

Tabela 4. Parâmetros de dinâmica da comunidade lenhosa (DAP $\geq 10 \mathrm{~cm}$ ) na mata de galeria do Gama nos períodos de 1985 a 1988, 1988 a 1991, 1991 a 1994, 1994 a 1999, 1999 a 2004 e 1985 a 2004, Fazenda Água Limpa, Brasília, DF. (m = taxa de mortalidade média anual, $\mathrm{R}=$ taxa de recrutamento médio anual, $\mathrm{T}_{1 / 2}=$ tempo de meia vida, $\mathrm{T}_{2}=$ tempo de duplicação, $\mathrm{R}=$ rotatividade, $\mathrm{E}=$ estabilidade $\mathrm{e}$ Tro $=$ taxa de rotatividade) .

Table 4. Parameters of the dynamic of the woody community (DAP $\geq 10 \mathrm{~cm}$ ) at the Gama gallery forest from 1985 to 1988 , 1988 to 1991, 1991 to 1999, 1999 to 2004 and 1985 to 2004. Água Limpa farm, Brasília, Brasil. (m = average annual mortality rate, $\mathrm{R}=$ average annual recruiting rate, $\mathrm{T}_{1 / 2}=$ half live, $\mathrm{T}_{2}=$ doubling time, $\mathrm{R}=$ turnover, $\mathrm{E}=$ stability and Tro $=$ turnover rate).

\begin{tabular}{lcccccc}
\hline & \multicolumn{7}{c}{ Períodos } \\
\cline { 2 - 7 } Parâmetros & 1985 a 1988 & 1988 a 1991 & 1991 a 1994 & 1994 a 1999* & 1999 a 2004 & 1985 a 2004 \\
\hline $\mathrm{m}\left(\%\right.$ ano $\left.^{-1}\right)$ & 3,56 & 3,06 & 3,50 & 4,15 & 1,64 & 2,87 \\
$\mathrm{R}\left(\%\right.$ ano $\left.^{-1}\right)$ & 3,00 & 3,07 & 2,46 & 2,21 & 2,18 & 2,08 \\
$\mathrm{~T}_{1 / 2}($ anos $)$ & 19,46 & 22,67 & 19,81 & 16,72 & 42,16 & 24,13 \\
$\mathrm{~T}_{2}$ (anos) & 23,11 & 22,60 & 28,16 & 31,42 & 31,84 & 33,30 \\
$\mathrm{R}($ anos) & 21,29 & 22,64 & 23,98 & 24,07 & 37,00 & 28,71 \\
$\mathrm{E}($ anos) & 3,64 & 0,08 & 8,35 & 14,70 & 10,32 & 9,17 \\
Tro $\left(\%\right.$ ano $\left.^{-1}\right)$ & 3,28 & 3,06 & 2,98 & 3,18 & 1,91 & 2,48 \\
\hline
\end{tabular}

* 1997 a 1998: Período de maior intensidade de El Niño desde 1983 (fonte: Slik 2004).

* 1997 to 1998: period of greater intensity of el ninho since 1983 (source: Slik 2004).

De acordo com o modelo logarítmico, o tempo de duplicação da comunidade arbórea da mata de galeria do Gama no período de 1985 a 2004 é 33,30 anos, variando de 22 a 33 anos para os seis períodos analisados (tabela 4).

Considerando-se todas as espécies e indivíduos da comunidade, entre 1985 e 2004, registrou-se uma diferença de $0,79 \%$ em favor da mortalidade. Como consequiência, o tempo de duplicação (33 anos) foi superior à meia-vida (24 anos). A rotatividade da mata foi de 28,71 anos e a estabilidade calculada em 9,17 anos (tabela 4).

Cerca de $92 \%$ de todas as espécies amostradas entre 1985 e 2004 apresentaram mortalidade, recrutamento ou ambos (tabela 5). Analisando as espécies com pelo menos 30 indivíduos em 1985 (tabela 5), verificou-se que a taxa de mortalidade para o período de 1985-2004 variou de $0,68 \%$ ano $^{-1}$ para Copaifera langsdorffii, uma espécie emergente (Felfili 1997c) até $12,12 \%$ ano $^{-1}$ para Piptocarpha macropoda (uma espécie pioneira colonizadora de clareiras, Felfili 1997c). A meia vida das espécies mais abundantes variou de 5 anos para $P$. macropoda até 101 anos para $C$. langsdorffii (tabela 5). Apesar da espécie $P$. macropoda apresentar a maior taxa de mortalidade na mata estudada, seu incremento sempre esteve acima da média da comunidade em todos os períodos analisados. 
Tabela 5. Parâmetros de dinâmica das populações das espécies com mais de 30 indivíduos em 1985 da comunidade lenhosa da mata de galeria do Gama no período de 1985 a 2004, Fazenda Água Limpa, Brasília, DF. ( $N$ = número de indivíduos em $1985, \mathrm{~m}=$ taxa de mortalidade média anual, $\mathrm{r}=$ taxa de recrutamento médio anual, $\mathrm{t}_{1 / 2}=$ tempo de meia vida e $\mathrm{t}_{2}=$ tempo de duplicação).

Table 5. Parameters of the dynamic of the populations with more than 30 individuals in 1985 in the woody community of the Gama gallery forest, Água Limpa farm, Brasília, Brazil. ( $N=$ number of individuals in $1985, \mathrm{~m}=$ average annual mortality rate, $\mathrm{r}=$ average annual recruiting rate, $\mathrm{t}_{1 / 2}=$ half live, $\mathrm{t}_{2}=$ doubling time).

\begin{tabular}{lrccrrr}
\hline Espécies & $N$ & $\begin{array}{c}\mathrm{m} \\
\left(\% \mathrm{ano}^{-1}\right)\end{array}$ & $\begin{array}{c}\mathrm{r} \\
\left(\% \mathrm{ano}^{-1}\right)\end{array}$ & $\begin{array}{c}\mathrm{t}_{1 / 2} \\
(\mathrm{anos})\end{array}$ & $\begin{array}{c}\mathrm{t}_{2} \\
(\mathrm{anos})\end{array}$ & $\begin{array}{c}\text { Tro } \\
\left(\% \text { ano }^{-1}\right)\end{array}$ \\
\hline Amaioua guianensis & 104 & 3,35 & 3,65 & 20,67 & 19,00 & 3,50 \\
Aspidosperma parvifolium & 92 & 1,75 & 0,54 & 39,65 & 127,63 & 1,15 \\
Callisthene major & 43 & 1,72 & 0,69 & 40,25 & 100,83 & 1,20 \\
Cheiloclinium cognatum & 102 & 2,13 & 1,81 & 32,48 & 38,19 & 1,97 \\
Copaifera langsdorffii & 74 & 0,68 & 0,79 & 101,56 & 87,63 & 0,74 \\
Cryptocaria aschersoniana & 48 & 1,97 & 1,68 & 35,15 & 41,36 & 1,82 \\
Cupania vernalis & 31 & 9,60 & 1,07 & 7,22 & 64,68 & 5,34 \\
Emmotum nitens & 37 & 2,28 & 0,14 & 30,42 & 493,84 & 1,21 \\
Guatteria sellowiana & 104 & 2,64 & 1,56 & 26,27 & 44,31 & 2,10 \\
Lamanonia ternata & 94 & 3,12 & 0,06 & 22,24 & 1244,53 & 1,59 \\
Licania apetala & 100 & 1,24 & 1,46 & 55,87 & 47,44 & 1,35 \\
Matayba guianensis & 32 & 2,74 & 1,91 & 25,26 & 36,29 & 2,33 \\
Maytenus alaternoides & 41 & 4,05 & 2,74 & 17,12 & 25,30 & 3,39 \\
Metrodorea pubescens & 116 & 2,16 & 1,73 & 32,14 & 40,17 & 1,94 \\
Nectandra mollis & 72 & 1,61 & 0,75 & 42,99 & 92,63 & 1,18 \\
Ouratea castaneifolia & 38 & 3,65 & 1,85 & 19,00 & 37,48 & 2,75 \\
Piptocarpha macropoda & 12,12 & 1,33 & 5,72 & 52,12 & 6,72 \\
Protium heptaphyllum & 30 & 3,65 & 2,75 & 19,00 & 25,21 & 3,20 \\
Salacia elliptica & 86 & 2,40 & 4,23 & 28,83 & 16,39 & 3,32 \\
Tapirira guianensis & 30 & 3,22 & 2,79 & 21,56 & 24,82 & 3,00 \\
\hline
\end{tabular}

A taxa de recrutamento para o período de 1985-2004 variou de $0,06 \%$ ano $^{-1}$ para Lamanonia ternata a $4,23 \%$ ano $^{-1}$ para Salacia elliptica (tabela 5). O tempo de duplicação das espécies Lamanonia ternata e Emmotum nitens foi elevado (1244 e 494 anos, respectivamente) devido à baixa taxa de recrutamento observado para essas espécies $\left(0,06\right.$ e $0,14 \%$ ano $^{-1}$, respectivamente). Amaioua guianensis foi a espécie que apresentou a segunda maior taxa de recrutamento $\left(3,65 \%\right.$ ano $\left.^{-1}\right)$, entretanto sua taxa de mortalidade também foi elevada $\left(3,35 \%\right.$ ano $\left.^{-1}\right)$.

\section{Discussão}

Incremento - $\mathrm{O}$ incremento periódico anual (IPA) da comunidade arbórea com DAP $\geq 10 \mathrm{~cm}$ da mata de galeria do $\operatorname{Gama}\left(0,22 \mathrm{~cm} \mathrm{ano}^{-1}\right)$ no período de 1985 a 2004 foi similar ao encontrado em avaliações anteriores da mata em questão $\left(0,25 \mathrm{~cm}^{2} \mathrm{ano}^{-1}\right.$ no período de 1985 a 1991 , Felfili 1995; e 0,22 cm ano-1 entre 1985 a 1999, Salgado 2003) para intervalos de 6 e 14 anos, respectivamente indicando que o ritmo de crescimento da floresta está se mantendo ou seja, provavelmente a proporção de espécies clímax, de crescimento mais lento, versus espécies pioneiras, de crescimento mais rápido, está estável nos 19 anos de estudo. Estes valores estão próximos dos encontrados na floresta do Vale do Véu da Noiva, no Parque Nacional da Chapada dos Guimarães $\left(0,21 \mathrm{~cm}^{2} \mathrm{ano}^{-1}\right.$, Pinto \& Hay 2005), e daqueles encontrados por Carvalho et al. 2004 em área não perturbada de floresta amazônica $\left(0,22 \mathrm{~cm} \mathrm{ano}^{-1}\right)$, por Silva et al. (1996) na Floresta Nacional do Tapajós $\left(0,20 \mathrm{~cm}^{2} \mathrm{ano}^{-1}\right)$ e por Lieberman et al (1985) para espécies arbóreas de uma floresta tropical na Costa Rica $\left(0,265 \mathrm{~cm} \mathrm{ano}^{-1}\right)$. Ou seja, taxas 
neste patamar parecem ser típicas de florestas tropicais vinculadas à ambientes úmidos e não perturbados. Valores mais elevados foram observados por Pulz (1998) em remanescente de floresta semidecídua montana em Minas Gerais $\left(0,457 \mathrm{~cm}^{a n o}{ }^{-1}\right)$, por Silva et al. (1995) em floresta tropical Amazônica 13 anos após corte experimental $\left(0,3 \mathrm{~cm}^{2} \mathrm{ano}^{-1}\right)$ e por Lang \& Knight (1983) em floresta tropical em Barro do Colorado $\left(0,9 \mathrm{~cm}\right.$ ano $\left.^{-1}\right)$. Estas taxas mais elevadas parecem ser vinculadas à ambientes perturbados (Amazônia sob exploração), com predomínio de espécies pioneiras, adaptadas a ambientes mais abertos (floresta semidecídua) e com efeito de borda, como é o caso da Ilha de Barrro Colorado.

O aumento do incremento com o DAP também foi verificado por Felfili (1995) para a mesma mata, no intervalo de 1985 a 1991. As taxas de crescimento nas florestas tropicais estudadas por Vieira et al. (2004) na Amazônia brasileira também foram dependentes do tamanho das árvores, com os indivíduos maiores apresentando as mais altas taxas de crescimento absoluto. De acordo com Hubbell et al. (1999), esta tendência de indivíduos maiores apresentarem maior incremento em diâmetro está relacionada com a maior atividade fotossintética desses indivíduos que, geralmente, dominam o dossel da floresta. Felfili (1995) enfatiza que devido à estrutura das florestas tropicais, onde a maioria dos indivíduos está concentrada nas menores classes de diâmetro, as médias de incremento nas maiores classes de diâmetro vêm de um número muito menor de árvores, resultando em informações que podem não representar a realidade da comunidade. De modo que as taxas encontradas para as classes de diâmetro até $52 \mathrm{~cm}$ (figura 1) representam os padrões de $95 \%$ da comunidade e são, portanto, bastante representativos.

De acordo com Felfili (2000), que fez uma revisão de vários trabalhos com matas de galeria, o incremento diamétrico médio para árvores adultas nas matas de galeria está na ordem de $0,30 \mathrm{~cm}$ de diâmetro por ano, similar a outras florestas tropicais conforme a mesma autora. As espécies Cryptocaria aschersoniana, Piptocarpha macropoda, Protium heptaphyllum e Tapirira guianensis que, nesse estudo, apresentaram incremento médio anual em diâmetro superior ao da comunidade (IPA $=0,22 \mathrm{~cm} \mathrm{ano}^{-1}$ ) para todos os períodos analisados, são exemplos de espécies lenhosas que crescem rápido, em média $0,5 \mathrm{~cm}$ de diâmetro por ano, em condições naturais, como apontadas por Felfili (2000). De acordo com Felfili (1997b), que fez uma comparação de ritmos de crescimento das espécies desta mata com os ritmos apresentados pelas mesmas espécies em outra mata de galeria perturbada por fogo na mesma bacia hidrográfica, C. aschersoniana e T. guianensis são espécies emergentes não pioneiras que toleram sombra. As demais espécies são dominantes, sendo que $P$. macropoda é pioneira e $P$. heptaphyllum não pioneira tolerante à sombra (Felfili 1997c).

Em geral, espécies que demandam luz e as que ocupam o dossel tendem a apresentar maiores taxas de incremento conforme foi constado por Felfili (1995) assim como as diferenças nos gradientes de luz foram estudadas por Felfili \& Abreu (1999). Assim, o rápido crescimento das espécies pioneiras possibilita que este grupo apresente, temporariamente, certa vantagem competitiva em relação às espécies de estágio secundário da sucessão, permitindo que elas alcancem a maturidade reprodutiva antes de serem excluídas pela competição conforme sugere Pinto $\&$ Hay (2005) estudando uma mata de galeria na Chapada dos Guimarães que apresenta várias espécies comuns com esta mata. Conforme Salgado (2003) confirmado pelos resultados deste estudo, as espécies não pioneiras tolerantes à sombra estão sendo favorecidas neste estágio de sucessão da comunidade da mata em questão em detrimento das espécies que ocupam os estratos superiores do dossel.

Mortalidade e recrutamento - As taxas médias anuais de mortalidade de $3,56 \%, 3,06 \%, 3,50 \%$ e $4,15 \%$ obtidas para a comunidade arbórea com DAP $\geq 10 \mathrm{~cm}$ da mata de galeria do Gama para os períodos de 1985 a 1988, 1988 a 1991, 1991 a 1994 e 1994 a 1999 são altas se comparadas aos valores de $2,68 \%$ ano $^{-1}$ em uma floresta de galeria em vale na Chapada dos Guimarães no Mato Grosso (Pinto \& Hay 2005), de 2,2\% ano ${ }^{-1}$, (Silva et al. 1995) e $2,5 \%$ ano $^{-1}$ (Korning \& Balslev 1994b) na floresta amazônica, de 1,62\% ano $^{-1}$ na mata atlântica (Pedroni 2001) e em outras florestas tropicais (Lieberman \& Lieberman 1987 (La Selva na Costa Rica), Swaine et al. 1987 (em Gana na África). Entretanto, as taxas de mortalidade para os períodos de 1999 a 2004 e 1985 a $2004\left(1,64 \%\right.$ ano $^{-1}$ e $2,87 \%$ ano $\left.^{-1}\right)$ representaram valores dentro da amplitude obtida ( 1 a $3 \%$ ) para a maioria dos estudos realizados na região tropical citados acima. Manokaran \& Kochummen (1987), Carey et al. (1994) e Condit et al. (1995) também encontraram marcante variação na mortalidade entre períodos sucessivos de monitoramento, indicando que as taxas de mortalidade podem ser muito variáveis temporalmente.

Os valores de mortalidade acima de $3 \%$ ano $^{-1}$ observados para a mata do Gama estão dentro do valor encontrada por Pulz (1998) em floresta semidecídua montana de Minas Gerais, no período de 1992 a 1996, de 3,6\% ano ${ }^{-1}$; por Cabral (1999), de 3,7\% ano $^{-1}$ em um 
fragmento de mata ciliar de Minas Gerais; por Nascimento et al. (1999), num fragmento de floresta estacional semidecídua, de 4,7\% ano ${ }^{-1}$ e por Marín et al. (2005) de 4,5 e 4,2\% ano $^{-1}$, respectivamente, para floresta decídua e mata de galeria em uma reserva florestal na Nicarágua durante período de 7 anos de estudo.

A elevada taxa de mortalidade em comparação com outras formações tropicais encontrada para a mata de galeria do Gama sugere uma comunidade bastante dinâmica, fato que pode ser confirmado pela alta taxa de rotatividade registrada nos períodos sucessivos de amostragem. De acordo com Felfili (2000), as taxas de mortalidade da comunidade, da ordem de $3 \%$ ano $^{-1}$, são mais intensas do que aquelas encontradas em largas formações contínuas como a floresta amazônica (da ordem de $1 \%$ ano $^{-1}$ em áreas não perturbadas), sendo que essa intensidade se deve, provavelmente, aos estresses mais intensos sofridos pelas matas de galeria, pois são estreitas e, conseqüentemente, mais suscetíveis, por exemplo, a ação de ventos, enchentes e queimadas. As oscilações nas taxas de mortalidade ao longo do tempo podem ser relativas a fatores como estresses climáticos, longevidade das árvores que ora compõe a comunidade, dentre outros que merecem ser estudados em detalhe.

Salgado (2003), estudando a dinâmica da comunidade e de populações da mata do Gama, sugeriu que o fator climático El Niño pode estar interferindo na dinâmica da floresta. A maior taxa de mortalidade foi observada em 1994 a 1999 sob a influência desse fenômeno, cujo registro mais intenso desde 1983 ocorreu entre 1997 a 1998 (Slik 2004). De acordo com Slik (2004), secas severas, tais como aquelas associadas com eventos de El Niño, podem afetar a composição de espécies arbóreas e a diversidade de florestas tropicais por meio da mortalidade desproporcional de certos grupos ecológicos e classes de tamanho dos indivíduos arbóreos, bem como pela mudança nas condições de luz do subosque da mata, que afetam o recrutamento e o crescimento de árvores pequenas e imaturas. Porém, os efeitos do El Niño sobre a região Centro-Oeste ainda não estão totalmente elucidados, sendo verificada uma tendência de chuvas acima da média (Salgado 2003). As chuvas trazem enxurradas que quebram os barrancos dos córregos ocasionando a queda de árvores assim como os fortes ventos (Felfili 1997 a,b) e a sua maior intensidade nos períodos de $E l$ Niño pode ser a causadora da acentuada mortalidade.

$\mathrm{Na}$ área amostrada a taxa de mortalidade da comunidade para o período de 19 anos (figura 2) foi mais acentuada nas menores classes de diâmetro (10 a $27 \mathrm{~cm}$ ), onde se concentram cerca de $77 \%$ dos indivíduos, fato verificado por Felfili (1995) para a mata em questão, o que evidencia que a competição por recursos (espaço, luz, nutrientes, água) é maior para os indivíduos menores do que para aqueles pertencentes às classes subseqüentes. Rankin-de-Merona et al. (1990), amostrando árvores com DAP $\geq 10 \mathrm{~cm}$ de uma floresta da Amazônia brasileira, encontraram maior proporção de árvores mortas entre os indivíduos das menores classes diamétricas, acompanhando o padrão de distribuição de abundância. Hubbell \& Foster (1990), estudando árvores a partir de $1 \mathrm{~cm}$ de diâmetro, encontraram maior mortalidade entre árvores com DAP $\geq 16 \mathrm{~cm}$ em Barro Colorado no Panamá. Esses autores atribuíram esta mortalidade ao período de seca prolongada causada pelo fenômeno El Niño de 1983, que afetou principalmente as espécies típicas de locais mais úmidos. Essa mortalidade nas menores classes sugere também que a mata pode estar atingindo um estágio de maturidade na silvigênese onde as grandes árvores se estabelecem, sombreiam o ambiente e o tornam desfavoráveis para as espécies de menor porte que demandam luz.

A meia-vida de 24 anos, observada na mata do Gama para o período de 1985 a 2004, está abaixo dos valores encontrados para florestas neotropicais que, segundo Hartshorn (1990) variam de 34 a 68 anos. Isto indica que a comunidade arbórea estudada apresenta elevada dinâmica, com taxa média anual de rotatividade de $2,48 \%$ ano $^{-1}$ e rotatividade de 28,71 anos (isto é, o tempo de reposição do número de indivíduos mortos num intervalo de tempo é de cerca de 29 anos), similar às florestas mais dinâmicas como em Barro Colorado (Hubbell \& Foster 1990) com 22,5 anos, como um fragmento de mata ciliar em Minas Gerais (Cabral 1999), com 26,82 anos e em floresta de galeria no Vale do Véu da Noiva no Mato Grosso (Pinto \& Hay 2005), com 23,61 anos.

As taxas de recrutamento médio anual estiveram na mesma amplitude de valor registrado por Pinto \& Hay (2005) na floresta de Vale do Véu da Noiva no Mato Grosso (3,25\% ano $\left.{ }^{-1}\right)$, por Silva et al. (1995) em áreas da floresta amazônica $\left(3,1 \%\right.$ ano $\left.^{-1}\right)$, por Cabral (1999) em fragmento de mata ciliar $\left(2,03 \%\right.$ ano $\left.^{-1}\right)$, por Pedroni (2001) em mata atlântica $\left(2,66 \%\right.$ ano $\left.^{-1}\right)$, por Lieberman \& Lieberman (1987) em florestas tropicais da América Central $\left(2,03 \%\right.$ ano $\left.^{-1}\right)$ e foram inferiores à encontrada por por Nascimento et al. (1999) num fragmento de floresta estacional semidecídua $\left(9,6 \%\right.$ ano $\left.^{-1}\right)$ e por Hubbell \& Foster (1990) em Barro Colorado, no Panamá $\left(4,48 \%\right.$ ano $\left.^{-1}\right)$.

A mata estudada apresentou maior mortalidade que recrutamento para a maioria dos períodos de amostragem, exceto nos intervalos de 1988 a 1991 e 1999 a 2004, confirmando a tendência encontrada por Felfili (1993, 
1995) que verificou comportamento similar a vários estudos em florestas tropicais (Lang \& Knight 1983, Manokaran \& Kochummen 1987, Swaine et al. 1987). Manokaran \& Kochummen (1987) encontraram variação no balanço entre mortalidade e recrutamento em períodos de observação sucessivos, sendo que, durante os 10 primeiros anos, a mortalidade superou o recrutamento e no período seguinte, houve um balanço entre as duas taxas. Após um período com elevada taxa de mortalidade, ocorre abertura de espaço para que, em seguida, ocorra um recrutamento mais intenso, caracterizando a floresta como um sistema dinâmico, que apresenta mudanças contínuas ao longo do tempo em sua estrutura e posição (Felfili 1995).

A forte correlação positiva entre mortalidade, recrutamento e densidade das espécies sugere que o elevado recrutamento das espécies abundantes tende a ser compensado por sua alta mortalidade, mantendo assim sua dominância, correlação também verificada por Felfili (1995). As espécies que compõem a comunidade apresentam distintas taxas de recrutamento, mortalidade e incremento, que parecem estar relacionadas aos grupos funcionais a que pertencem e a seu posicionamento no dossel.

A comunidade da mata de galeria do Ribeirão do Gama, no trecho estudado, é dinâmica, com $92 \%$ de todas as espécies amostradas apresentando mortalidade, recrutamento ou ambos. Entretanto, apresentou estabilidade na composição florística durante os 19 anos de estudo, uma vez que $83 \%$ do total de espécies encontrado na mata ocorreram em todos os períodos analisados (figura 1). As taxas de incremento, mortalidade e recrutamento intensas indicam uma comunidade mais dinâmica do que as extensas florestas tropicais úmidas, provavelmente devido aos estresses sofridos pelo efeito da borda com a vegetação de campo e de cerrado e pelas inundações periódicas.

Agradecimentos - somos gratas à equipe de campo, Sr. Newton Rodrigues, Kennya M. O. Ramos, Patrícia Dias Avelino, Vanessa Tunholi e Juliana Martins pelo auxílio na coleta dos dados. Ao CNPq (130057/2003-7) pela concessão da bolsa de mestrado à primeira autora, de bolsas do Programa PELD, de iniciação científica e de produtividade em pesquisas para a segunda autora.

\section{Referências bibliográficas}

CABRAL, V.A.R. 1999. Dinâmica de um fragmento de mata ciliar do rio Grande em Bom Sucesso - Minas Gerais. Dissertação de mestrado. Universidade Federal de Lavras, Lavras.
CAREY, E.V., BROWN, S., GILLESPIE, A.J.R. \& LUGO, A.E. 1994. Tree mortality in mature lowland moist and tropical lower moist forests of Venezuela. Biotropica 26:255-265.

CARVALHO, J.O.P., SILVA, J.N.M. \& LOPES, J.C.A. 2004. Growth rate of a terra firme rain forest in Brazilian Amazonia over an eight-year period in response to logging. Acta Amazonica 34:209-217.

CONDIT, R., HUBBELL, S.P. \& FOSTER, R.B. 1995. Mortality rates of 205 neotropical tree and shrub species and the impact of a severe drought. Ecological Monographs 65:419-439.

DAMASCENO-JUNIOR, G.D., SEMIR, J., SANTOS, F.A. M. \& LEITÃO-FILHO, H.F. 2004. Tree mortality in a riparian forest at Rio Paraguai, Pantanal, Brazil, after an extreme flooding. Acta Botanica Brasilica 18:839-846.

EITEN, G. 1984. Vegetation of Brasilia. Phytocoenologia 12:271-292.

FELFILI, J.M. 1994. Floristic composition and phytosociology of the gallery forest alongside the Gama stream in Brasília, DF, Brazil. Revista Brasileira de Botânica 17:1-11.

FELFILI, J.M. 1995a. Growth, recruitment and mortality in Gama gallery forest in central Brazil over a six-year period (1985-1991). Journal of Tropical Ecology 11:67-83.

FELFILI, J.M. 1995b. Diversity, structure, and dynamics of a gallery forest in central Brazil. Vegetatio 117:1-15.

FELFILI, J.M. 1997a. Comparison of the dynamics of two gallery forests in Central Brasil. In Proceedings: International symposium on assessment and monitoring of forests in tropical dry regions with special reference to gallery forests. (J. Imanãs-Encinas \& C. Kleinn, orgs.). University of Brasilia. Brasília. p.115-124.

FELFILI, J.M. 1997b. Dynamics of the natural regeneration in the Gama gallery forest in central Brazil. Forest Ecology and Management 91:235-245.

FELFILI, J.M. 1997c. Diameter and height distributions in the gallery forest tree community and some of its main species in central Brazil over a six-year period (19851991). Revista Brasileira de Botânica 20:155-162.

FELFILI, J.M. \& ABREU, H.M. 1999. Regeneração natural de Roupala montana Aubl., Piptocarpha macropoda Back. e Persea fusca Mez. em quatro condições ambientais na mata de galeria do Gama-DF. Cerne 5:125-132.

FELFILI, J.M. 2000. Crescimento, recrutamento e mortalidade nas matas de galeria do Planalto Central. In Tópicos atuais em botânica (T.B. Cavalcanti \& B.M.T Walter, orgs.). Embrapa, Brasília, p.152-158.

FELFILI, J.M. 2001. Principais fisionomias do Espigão Mestre do São Francisco. In Biogeografia do Bioma Cerrado - Estudo fitofisionômico na Chapada do Espigão Mestre do São Francisco (J.M. Felfili \& M.C. Silva Júnior, orgs.). Ministério do Meio Ambiente, Brasília, p.18-30.

FINGER, C.A.G. 1992. Fundamentos de biometria florestal. CEPEF-FATEC, Santa Maria. 
HARTSHORN, G.S. 1990. An overview of neotropical forest dynamics. In Four neotropical rainforests (A.H. Gentry, ed.). Yale University Press, New Haven, p.585-600.

HUBBELL, S.P. \& FOSTER, R.B. 1990. Structure, dynamics and equilibrium status of old-growth forest on Barro Colorado Island. In Four neotropical rainforests (A.H. Gentry, ed.). Yale University Press, New Haven, p.522-542.

HUBBELL, S.P., FOSTER, R.B., O'BRIEN, S.T., HARMS, K.E., CONDIT, R., WESCHSLER, B., WRIGTH, S. \& LOO de LAO, S. 1999. Light-gap disturbances, recruitment limitation, and tree diversity in a Neotropical forest. Science 283:554-557.

KELLMAN, M. \& MEAVE, J. 1997. Fire in the tropical gallery forests of Belize. Journal of Biogeography 24:23-34.

KORNING, J. \& BALSLEV, H. 1994a. Growth and mortality of trees in Amazonian tropical rain forest in Ecuador. Journal of Vegetation Science 4:77-86.

KORNING, J. \& BALSLEV, H. 1994b. Growth rates and mortality patterns of tropical lowland tree species and relation to forest structure in Amazonian Ecuador. Journal of Tropical Ecology 10:151-166.

LANG, G.E. \& KNIGHT, D.H. 1983. Tree growth, mortality, recruitment, and canopy gap formation during a 10-year period in a tropical moist forest. Ecology 64:1075-1080.

LIEBERMAN, D. \& LIEBERMAN, M. 1987. Forest tree growth and dynamics at La Selva, Costa Rica (19691982). Journal of Tropical Ecology 3:347-358.

LIEBERMAN, D., LIEBERMAN, M., PERALTA, R. \& HARTSHORN, G.S. 1985. Mortality patterns and stand turnover rates in a wet tropical forest in Costa Rica. Journal of Ecology 73:915-924.

LOPES, S.F. \& SCHIAVINI, I. 2007. Dinâmica da comunidade arbórea de mata de galeria da Estação Ecológica do Panga, Minas Gerais, Brasil. Acta Botânica Brasilica 21:249-261.

MANOKARAN, N. \& KOCHUMMEN, M. 1987. Recruitment, growth and mortality of tree species in a lowland dipterocarp forest in Peninsular Malaysia. Journal of Tropical Ecology 3:315-330.

MARÍN, G.C., NYGARD, R., RIVAS, B.G. \& ODEN, P.C. 2005. Stand dynamics and basal area change in a tropical dry forest reserve in Nicaragua. Forest Ecology and Management 208:63-75.

NASCIMENTO, H.E.M, DIAS, A.S., TABANEZ, A.A.J. \& VIANA, V.M. 1999. Estrutura e dinâmica de populações arbóreas de um fragmento de floresta estacional semidecidual na região de Piracicaba, SP. Revista Brasileira de Biologia 59:329-342.

NIMER, E. 1989. Climatologia do Brasil. IBGE, Departamento de Recursos Naturais e Estudos Ambientais, Rio de Janeiro.

OLIVEIRA-FILHO, A.T. \& RATTER, J.A. 1995. A study of the origin of central brazilian forests by the analysis of plant species distribution pattern. Edinburgh Journal of Botany 52:141-194.
PEDRONI, F. 2001. Aspectos da estrutura e dinâmica da comunidade arbórea na mata atlântica de planície e encosta em Picinguaba, Ubatuba, SP. Tese de doutorado, Universidade Estadual de Campinas, Campinas.

PINTO, J.R.R. \& HAY, J.D.V. 2005. Mudanças florísticas e estruturais na comunidade arbórea de uma floresta de vale no Parque Nacional da Chapada dos Guimarães, Mato Grosso, Brasil. Revista Brasileira de Botânica, 28:523-539.

PULZ, F.A. 1998. Estudo da dinâmica e a modelagem da estrutura diamétrica de uma floresta semidecídua Montana na região de Lavras-MG. Dissertação de mestrado, Universidade Federal de Lavras, Lavras.

RANKIN-DE-MERONA, J.M., HUTCHINGS, H.R.W. \& LOVEJOY, T.E. 1990. Tree mortality and recruitment over a five-year period in undisturbed upland rainforest of the Central Amazonian. In Four neotropical rainforests (A.H. Gentry, ed.). Yale University Press, New Haven, p.573-584.

RIBEIRO, J.F. \& WALTER, B.M.T. 2001. As matas de galeria no contexto do bioma Cerrado. In Cerrado caracterização e recuperação de matas de galeria (J.F. Ribeiro, C.E.L. Fonseca \& J.C. Sousa-Silva, eds.). Embrapa-CPAC, Planaltina, p.29-47.

SALGADO, M.A.S. 2003. Dinâmica de comunidade e de populações da mata de galeria do Gama, Distrito Federal. Dissertação de mestrado, Universidade de Brasília, Brasília, DF.

SILVA, J.N.M., CARVALHO, J.O.P., LOPES, J.C.A., ALMEIDA, B.F., COSTA, D.H.M., OLIVEIRA, L.C., VANCLAY, J.K. \& SKOVSGAARD, J.P. 1995. Growth and yield of a tropical rain forest in the Brazilian Amazon 13 years after logging. Forest Ecology and Management 71:267-274.

SILVA, J.N.M., CARVALHO, J.O.P., LOPES, J.C.A., OLIVEIRA, R.P. \& OLIVERIA, L.C. 1996. Growth and yield studies in the Tapajós region, Central Brazilian Amazon. Commonwealth Forestry Review 75:3325-3329.

SLIK, J.W.F. 2004. El Niño droughts and their effects on tree species composition and diversity in tropical rain forests. Oecologia 141:114-120.

SWAINE, M.D. \& LIEBERMAN, D. 1987. Note on the calculation of mortality rates. Journal of Tropical Ecology 3:ii-iii.

SWAINE, M.D., HALL, J.B. \& ALEXANDER, I.J. 1987. Tree population dynamics at Kade, Ghana (1968-1982). Journal of Tropical Ecology 3:331-345.

VIEIRA, S., CAMARGO, P.B., SELHORST, D., SILVA, R., HUTYRA, L., CHAMBERS, J.Q., BROWN, I.F., HIGUCHI, J.S., WOFSY, S.C., TRUMBORE, S.E. \& MARTINELLI, L.A. 2004. Forest structure and carbon dynamics in Amazonian tropical rain forests. Oecologia 140:468-479.

ZAR, J.H. 1999. Biostatistical analysis. Prentice-Hall, New Jersey. 